\section{MS7-P7 X-ray crystallographic and photophysical studies of DNA i-motifs}

Sarah P. Gurung ${ }^{1}$, James P. Hall ${ }^{1,2}$, Graeme Winter ${ }^{1}$, John A. Brazier $^{3}$, Rohanah Hussain ${ }^{1}$, Giuliano Siligardi ${ }^{1}$, Thomas Sorensen ${ }^{1}$, Christine J. Cardin²,4

1. Diamond Light Source, Harwell Science and Innovation Campus, Didcot, UK

2. Department of Chemistry, University of Reading, Reading, UK

3. School of Pharmacy, University of Reading, Reading, UK

4. Dynamic Structural Sciences, Research Complex at Harwell,

Harwell Science and Innovation Campus, Didcot, UK

email: sarah.gurung@diamond.ac.uk

An i-motif is a four stranded structure made of cytosine-rich DNA sequences. Its sequence is usually in the format of $\mathrm{C}_{2-5} \mathrm{~L}_{1-9} \mathrm{C}_{2-5} \mathrm{~L}_{1-9} \mathrm{C}_{2-5} \mathrm{~L}_{1-9} \mathrm{C}_{2-5}$, where $\mathrm{C}$ is cytosine and $\mathrm{L}^{2-5}$ represents any other base. The conformational change from the C-rich single strand DNA to i-motif takes place between $\mathrm{pH} 5$ and 6.7. These acidic conditions help two parallel i-motif duplexes "zip" together in an antiparallel orientation by protonating N3 in cytosines to create hemiprotonated $\mathrm{C}^{-} \mathrm{C}^{+}$base pairs (fig. 1). ${ }^{1}$

The i-motif can form as either an inter- or an intramolecular structure. However, only six i-motif crystal structures have been reported on the NDB; all of which are tetramolecular, even though i-motifs in vivo would exist as unimolecular. The c-Myc, Bcl-2 and hTERT i-motifs are all unimolecular and are present in the promoter regions of their respective oncogenes. ${ }^{2}$ Like the guanine rich G-quadruplex, the presence of a cytosine rich sequence has also been detected within the promoter region of the human telomeric and centromeric DNA, making i-motifs an attractive subject for gene transcription modulation.

UV and synchrotron radiation CD (srCD; beamline B23 at Diamond Light Source) spectroscopy were used to study the structural stability of intramolecular i-motifs. Our results showed that i-motifs with shorter loop lengths exhibit the highest stability. ${ }^{3}$ Crystallisation trials based on these initial results will be discussed along with previously recorded i-motif crystals grown in new conditions. We will also be reporting the diffraction of $\mathrm{d}$ (CCCT) crystals at $0.68 \AA$ at beamline I02, illustrating the advances in modern-day DNA crystallography via synchrotron radiation. Combination of results from the mentioned instrumental approaches shows that these methods are actually complementary.

\section{References}

1. Gehring, K., Leroy, J. L. \& Gueron, M. A tetrameric DNA structure with protonated cytosine.cytosine base pairs. Nature 363, 561-565 (1993).

2. Phan, A. T. \& Mergny, J.-L. Human telomeric DNA: G-quadruplex, i-motif and Watson-Crick double helix. Nucleic acids research 30, 4618-25 (2002).

3. Gurung, S. P., Schwarz, C., Hall, J. P., Cardin C. J. \& Brazier, J. A. The importance of loop length in the stability of i-motif structures. Chem. Commun. 51, 5630-32 (2015).
(A)

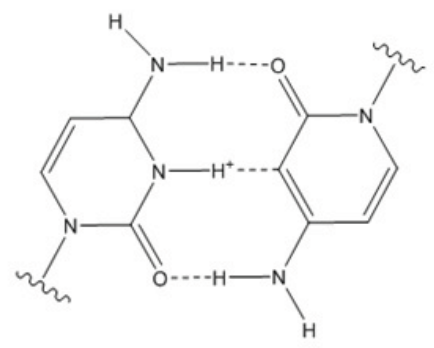

(B)
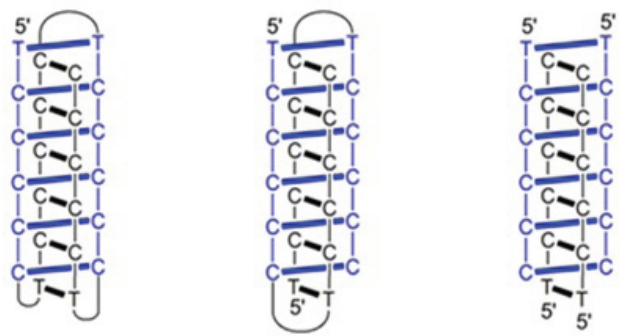

Figure 1. (A) $\mathrm{C}-\mathrm{C}^{+}$base pairing in i-motifs. (B) Schematic diagrams of unimolecular (left) bimolecular (middle) and tetramolecular (right) i-motifs.

Keywords: i-motif, DNA, srCD 